\title{
Communication
}

[Comunicação]

\section{Modified E-test by the addition of EDTA-Tris and dimethyl sulfoxide on the potentiation of the effects of some antimicrobials in Pseudomonas aeruginosa strains isolated from bovine mastitis}

\author{
[E-test modificado pela adição de Tris-EDTA e dimetilsulfóxido na potencialização do efeito de antimicrobianos em \\ linhagens de Pseudomonas aeruginosa isoladas de mastite bovina]
}

\author{
M.G. Ribeiro, A.K. Siqueira, H. Langoni, A.C. Paes, C. Victória, \\ A.V. Da Silva, F.J.P. Listoni, A.H. Souza \\ Departamento de Higiene Veterinária e Saúde Pública - FMVZ-UNESP \\ Caixa Postal 560 \\ 18618-000 - Botucatu, SP
}

Pseudomonas aeruginosa ( $P$. aeruginosa) present multiple drug resistance to conventional therapy and is considered an important agent in nosocomial infections (Paul et al., 1997). In domestic animals, $P$. aeruginosa is characterized as environmental agent in bovine mastitis. Bovine mammary infections have been associated with contamination of antimicrobial preparations used in intramammary therapy (both during lactation and in dry cow therapy), in water used in pre- or post-dipping procedures and in the cleaning procedures of milk equipment (Osborne et al., 1981; Radostits et al., 2000). Due to multiple drug resistance of $P$. aeruginosa strains, different chemical compounds have been investigated as potenciating drugs of antimicrobials effects. Ethylenediamine tetra-acetic acid (EDTA) cause damage in cell surfaces of the microorganisms, due to increase cell wall permeability to extracellular solutes. Tromethamine (Tris) buffer enhances EDTA effects, potentiating the action of some antimicrobials (Farca et al., 1993).

Dimethyl sulphoxide (DMSO) is a drug with more of 30 pharmacological actions described, present low toxicity, and has been used both in human and veterinary medicine in recent years. As EDTA-Tris, DMSO also increase cell wall permeability and has been used as an antibacterial, anti-fungic and anti-viral drug

Recebido para publicação em 14 de novembro de 2003 E-mail: mgribeiro@fmvz.unesp.br enhancer (Brayton, 1986). The objective of the present study was to investigate the enhancement effects on the minimal inhibitory concentrationsMIC of gentamicin, ciprofloxacin and norfloxacin in $P$. aeruginosa strains isolated from bovine mastitis, by addiction of EDTA-Tris or DMSO on E-Test medium.

Clinical isolates were obtained from 18 clinical and 12 subclinical cases identified at the Infectious Diseases of Domestic Animals and Research Nucleus on Mastitis-FMVZ-USP and FMVZ-UNESP/Botucatu, SP. Milk samples were cultured on defibrinated sheep blood agar $(5 \%)$ and MacConkey agar, incubated at $37^{\circ} \mathrm{C}$ for $72 \mathrm{~h}$. $P$. aeruginosa was classified according to macro-microscopical morphology and biochemical profile (Krieg and Holt, 1984). P. aeruginosa ATCC 10145 was used as the control.

All $P$. aeruginosa strains were submitted to standard MIC performed as described by the manufacturer $^{1}$, using gentamicin (0.016$256 \mathrm{mcg} / \mathrm{ml})$, ciprofloxacin $(0.002-32 \mathrm{mcg} / \mathrm{ml})$ and norfloxacin $(0.016-256 \mathrm{mcg} / \mathrm{ml})$. Thirty-one $P$. aeruginosa strains were simultaneously submitted to E-test modified by aseptically adding EDTA-Tris or DMSO to the original medium, using inoculum of standard densitiy and submitting the strains to the same antimicrobials describe above.

${ }^{1}$ Probac do Brasil 
The modified EDTA-Tris E-test was performed using solutions containing 250mmol/1 EDTA and $50 \mathrm{mmol} / 1$ tris, titrated to $\mathrm{pH} 8.0$ and sterilized (Farca et al., 1997). The modified DMSO E-test were produced by adding DMSO 5.0\%, after sterilization and cooling of Muller-Hinton medium (Ribeiro et al., 2001). MIC values in Etest were interpreted according to the American National Committee for Clinical and Laboratory Standards (NCCLS, 1999) The statistical analysis was performed using Kruskall-Wallis and Mann-Whitney tests $(\alpha=0.05)$ (Triola, 1999).
Table 1 presents the results of MIC values in standard E-test, and in E-test modified by the addition of DMSO or EDTA-Tris. In the standard E-test, 13 (41.9\%) strains were resistant to gentamicin. In modified methods using DMSO or EDTA-Tris, 12 of these 13 resistant strains were susceptible to gentamicin. All of 31 strains were susceptible to ciprofloxacin and norfloxacin both in standard and modified methods. Modified E-Tests showed statistically significant $(\mathrm{P}<0.05) \quad$ MIC reduction using gentamicin, ciprofloxacin and norfloxacin against $P$. aeruginosa strains.

Table 1. Minimal inhibitory concentration in Pseudomonas aeruginosa strains isolated from bovine mastitis in standard E-test and in modified method, by addition on EDTA-tris and dimethyl sulfoxide

\begin{tabular}{|c|c|c|c|c|c|c|c|c|c|}
\hline \multirow[b]{4}{*}{ Strain } & \multicolumn{9}{|c|}{ Antimicrobial } \\
\hline & \multicolumn{9}{|c|}{ Inhibition zone diameter - mm ( susceptibility) } \\
\hline & \multicolumn{3}{|c|}{ Standard E-test } & \multicolumn{3}{|c|}{ E-test with DMSO } & \multicolumn{3}{|c|}{ E-test with EDTA - tris } \\
\hline & $\mathrm{C}$ & $\mathrm{G}$ & $\mathrm{N}$ & $\mathrm{C}$ & $\mathrm{G}$ & $\mathrm{N}$ & $\mathrm{C}$ & $\mathrm{G}$ & $\mathrm{N}$ \\
\hline 1 & $0,125(\mathrm{~S})$ & $2(\mathrm{~S})$ & $1(\mathrm{~S})$ & $0,94(\mathrm{~S})$ & $3(\mathrm{~S})$ & $0,75(\mathrm{~S})$ & $0,25(\mathrm{~S})$ & $4(\mathrm{~S})$ & $1(\mathrm{~S})$ \\
\hline 2 & $0,125(\mathrm{~S})$ & $2(\mathrm{~S})$ & $0,25(\mathrm{~S})$ & $0,125(\mathrm{~S})$ & $3(\mathrm{~S})$ & $0,38(\mathrm{~S})$ & $0,125(\mathrm{~S})$ & $2(\mathrm{~S})$ & $0,75(\mathrm{~S})$ \\
\hline 3 & $0,094(\mathrm{~S})$ & $2(\mathrm{~S})$ & $0,5(\mathrm{~S})$ & $0,125(\mathrm{~S})$ & $3(\mathrm{~S})$ & $0,5(\mathrm{~S})$ & $0,125(\mathrm{~S})$ & $2(\mathrm{~S})$ & $0,38(\mathrm{~S})$ \\
\hline 4 & $0,38(\mathrm{~S})$ & $256(\mathrm{R})$ & $4(\mathrm{~S})$ & $0,125(\mathrm{~S})$ & $6(\mathrm{R})$ & $0,5(\mathrm{~S})$ & $0,125(\mathrm{~S})$ & $1,5(\mathrm{~S})$ & $0,5(\mathrm{~S})$ \\
\hline 5 & $0,125(\mathrm{~S})$ & $8(\mathrm{R})$ & $0,75(\mathrm{~S})$ & $0,094(\mathrm{~S})$ & $1,5(\mathrm{~S})$ & $0,38(\mathrm{~S})$ & $0,19(\mathrm{~S})$ & $1(\mathrm{~S})$ & $0,75(\mathrm{~S})$ \\
\hline 6 & $0,125(\mathrm{~S})$ & $6(\mathrm{R})$ & $1(\mathrm{~S})$ & $0,125(\mathrm{~S})$ & $3(\mathrm{~S})$ & $0,38(\mathrm{~S})$ & $0,19(\mathrm{~S})$ & $2(\mathrm{~S})$ & $0,38(\mathrm{~S})$ \\
\hline 7 & $0,19(\mathrm{~S})$ & $6(\mathrm{R})$ & $0,75(\mathrm{~S})$ & $0,125(\mathrm{~S})$ & $1,5(\mathrm{~S})$ & $0,38(\mathrm{~S})$ & $0,094(\mathrm{~S})$ & $1(\mathrm{~S})$ & $0,25(\mathrm{~S})$ \\
\hline 8 & $0,19(\mathrm{~S})$ & $2(\mathrm{~S})$ & $0,5(\mathrm{~S})$ & $0,094(\mathrm{~S})$ & $1,5(\mathrm{~S})$ & $0,38(\mathrm{~S})$ & $0,75(\mathrm{~S})$ & $2(\mathrm{~S})$ & $8(\mathrm{R})$ \\
\hline 9 & $0,125(\mathrm{~S})$ & $1(\mathrm{~S})$ & $0,38(\mathrm{~S})$ & $0,094(\mathrm{~S})$ & $3(\mathrm{~S})$ & $0,25(\mathrm{~S})$ & $0,047(\mathrm{~S})$ & $0,75(\mathrm{~S})$ & $0,094(\mathrm{~S})$ \\
\hline 10 & $0,094(\mathrm{~S})$ & $4(S)$ & $0,75(\mathrm{~S})$ & $0,064(\mathrm{~S})$ & $2(\mathrm{~S})$ & $0,38(\mathrm{~S})$ & $0,125(\mathrm{~S})$ & $1,5(\mathrm{~S})$ & $0,5(\mathrm{~S})$ \\
\hline 11 & $0,38(\mathrm{~S})$ & $3(\mathrm{~S})$ & $1,5(\mathrm{~S})$ & $0,19(\mathrm{~S})$ & $0,25(\mathrm{~S})$ & $0,75(\mathrm{~S})$ & $0,047(\mathrm{~S})$ & $1,5(\mathrm{~S})$ & $0,19(\mathrm{~S})$ \\
\hline 12 & $0,125(\mathrm{~S})$ & $3(\mathrm{~S})$ & $1(\mathrm{~S})$ & $0,125(\mathrm{~S})$ & $1,5(\mathrm{~S})$ & $0,75(\mathrm{~S})$ & $0,125(\mathrm{~S})$ & $0,75(\mathrm{~S})$ & 0,19 (S) \\
\hline 13 & $0,094(\mathrm{~S})$ & $1,5(\mathrm{~S})$ & $0,38(\mathrm{~S})$ & $0,125(\mathrm{~S})$ & $0,25(\mathrm{~S})$ & $0,75(\mathrm{~S})$ & $0,064(\mathrm{~S})$ & $1(\mathrm{~S})$ & $0,19(\mathrm{~S})$ \\
\hline 14 & $0,19(\mathrm{~S})$ & $3(\mathrm{~S})$ & $0,38(\mathrm{~S})$ & $0,125(\mathrm{~S})$ & $1(\mathrm{~S})$ & $0,75(\mathrm{~S})$ & $0,094(\mathrm{~S})$ & $0,5(\mathrm{~S})$ & $0,19(\mathrm{~S})$ \\
\hline 15 & $0,25(\mathrm{~S})$ & $6(\mathrm{R})$ & $1(\mathrm{~S})$ & $0,064(\mathrm{~S})$ & $0,047(\mathrm{~S})$ & $0,25(\mathrm{~S})$ & $0,094(\mathrm{~S})$ & $3(\mathrm{~S})$ & $0,25(\mathrm{~S})$ \\
\hline 16 & 0,19 (S) & $3(\mathrm{~S})$ & $1(\mathrm{~S})$ & $0,094(\mathrm{~S})$ & $0,5(\mathrm{~S})$ & $0,38(\mathrm{~S})$ & $0,125(\mathrm{~S})$ & $1,5(\mathrm{~S})$ & $0,25(\mathrm{~S})$ \\
\hline 17 & $0,38(\mathrm{~S})$ & $3(\mathrm{~S})$ & $0,5(\mathrm{~S})$ & $0,064(\mathrm{~S})$ & $0,064(\mathrm{~S})$ & $0,25(\mathrm{~S})$ & $0,064(\mathrm{~S})$ & $1(\mathrm{~S})$ & $0,19(\mathrm{~S})$ \\
\hline 18 & $0,19(\mathrm{~S})$ & $6(\mathrm{R})$ & $0,75(\mathrm{~S})$ & $0,094(\mathrm{~S})$ & $1(\mathrm{~S})$ & $0,38(\mathrm{~S})$ & $0,094(\mathrm{~S})$ & $1(\mathrm{~S})$ & $0,38(\mathrm{~S})$ \\
\hline 19 & $0,125(\mathrm{~S})$ & $8(\mathrm{R})$ & $0,75(\mathrm{~S})$ & $0,094(\mathrm{~S})$ & $2(\mathrm{~S})$ & $0,38(\mathrm{~S})$ & $0,047(\mathrm{~S})$ & $1(\mathrm{~S})$ & $0,19(\mathrm{~S})$ \\
\hline 20 & $0,125(\mathrm{~S})$ & $2(\mathrm{~S})$ & $0,5(\mathrm{~S})$ & $0,094(\mathrm{~S})$ & $3(\mathrm{~S})$ & $0,38(\mathrm{~S})$ & $0,094(\mathrm{~S})$ & $2(\mathrm{~S})$ & $0,38(\mathrm{~S})$ \\
\hline 21 & $0,19(\mathrm{~S})$ & $6(\mathrm{R})$ & $1(\mathrm{~S})$ & $0,19(\mathrm{~S})$ & $2(\mathrm{~S})$ & $0,75(\mathrm{~S})$ & $0,094(\mathrm{~S})$ & $1,5(\mathrm{~S})$ & $0,25(\mathrm{~S})$ \\
\hline 22 & $0,19(\mathrm{~S})$ & $3(\mathrm{~S})$ & $0,5(\mathrm{~S})$ & $0,094(\mathrm{~S})$ & $1(\mathrm{~S})$ & $0,5(\mathrm{~S})$ & $0,094(\mathrm{~S})$ & $1(\mathrm{~S})$ & $0,25(\mathrm{~S})$ \\
\hline 23 & $0,125(\mathrm{~S})$ & $3(\mathrm{~S})$ & $0,38(\mathrm{~S})$ & $0,094(\mathrm{~S})$ & $1,5(\mathrm{~S})$ & $0,5(\mathrm{~S})$ & $0,064(\mathrm{~S})$ & $1,5(\mathrm{~S})$ & $0,125(\mathrm{~S})$ \\
\hline 24 & $0,094(\mathrm{~S})$ & $2(\mathrm{~S})$ & $0,25(\mathrm{~S})$ & $0,047(\mathrm{~S})$ & $0,5(\mathrm{~S})$ & $0,38(\mathrm{~S})$ & $0,064(\mathrm{~S})$ & $3(\mathrm{~S})$ & $0,25(\mathrm{~S})$ \\
\hline 25 & $0,38(\mathrm{~S})$ & $12(\mathrm{R})$ & $0,75(\mathrm{~S})$ & $0,032(\mathrm{~S})$ & $1(\mathrm{~S})$ & $0,38(\mathrm{~S})$ & $0,064(\mathrm{~S})$ & $1(\mathrm{~S})$ & $0,19(\mathrm{~S})$ \\
\hline 26 & $0,064(\mathrm{~S})$ & $6(\mathrm{R})$ & $0,125(\mathrm{~S})$ & $0,032(\mathrm{~S})$ & $1,5(\mathrm{~S})$ & $0,125(\mathrm{~S})$ & $0,047(\mathrm{~S})$ & $1,5(\mathrm{~S})$ & $0,125(\mathrm{~S})$ \\
\hline 27 & $0,125(\mathrm{~S})$ & $2(\mathrm{~S})$ & $0,75(\mathrm{~S})$ & $0,094(\mathrm{~S})$ & $0,75(\mathrm{~S})$ & $0,25(\mathrm{~S})$ & $0,125(\mathrm{~S})$ & $0,75(\mathrm{~S})$ & $0,38(\mathrm{~S})$ \\
\hline 28 & $0,125(\mathrm{~S})$ & $2(\mathrm{~S})$ & $0,75(\mathrm{~S})$ & $0,032(\mathrm{~S})$ & $0,5(\mathrm{~S})$ & $0,19(\mathrm{~S})$ & $0,064(\mathrm{~S})$ & $0,38(\mathrm{~S})$ & $0,25(\mathrm{~S})$ \\
\hline 29 & $0,19(\mathrm{~S})$ & $4(\mathrm{~S})$ & $0,75(\mathrm{~S})$ & $0,094(\mathrm{~S})$ & $1,5(\mathrm{~S})$ & $0,5(\mathrm{~S})$ & $0,094(\mathrm{~S})$ & $2(\mathrm{~S})$ & $0,38(\mathrm{~S})$ \\
\hline 30 & $0,19(\mathrm{~S})$ & $3(\mathrm{~S})$ & $0,75(\mathrm{~S})$ & $0,094(\mathrm{~S})$ & $1(\mathrm{~S})$ & $0,38(\mathrm{~S})$ & $0,19(\mathrm{~S})$ & $2(\mathrm{~S})$ & $0,5(\mathrm{~S})$ \\
\hline 31 & $0,125(\mathrm{~S})$ & $6(\mathrm{R})$ & $0,75(\mathrm{~S})$ & $0,094(\mathrm{~S})$ & $1(\mathrm{~S})$ & $0,5(\mathrm{~S})$ & $0,094(\mathrm{~S})$ & $1,5(\mathrm{~S})$ & $0,25(\mathrm{~S})$ \\
\hline median & 0.125 & 3.000 & 0.750 & 0.094 & 1.500 & 0.380 & 0.094 & 1.500 & 0.250 \\
\hline Standard error & 0.02 & 10.199 & 0.149 & 0.008 & 0.28 & 0.04 & 0.028 & 0.176 & 0.313 \\
\hline
\end{tabular}

Ciprofloxacin (C) range $(0.002-32 \mathrm{mcg} / \mathrm{ml})$; Gentamicin $(\mathrm{G})$ range $(0.016-256 \mathrm{mcg} / \mathrm{ml})$; Norfloxacin $(\mathrm{N})$ range $(0.016-$ $256 \mathrm{mcg} / \mathrm{ml}) ; \mathrm{S}=$ susceptible; $\mathrm{R}=$ resistant; $\mathrm{DMSO}=$ dimethyl sulfoxide; EDTA - tris= ethylene diamine tetra acetic acid tromethamine. 
Farca et al. (1993) showed in vitro potentiation of aminoglycosides and quinolones effects adding EDTA-Tris in E-test against $P$. aeruginosa, Proteus mirabilis and Escherichia coli strains. Ribeiro et al. (2001) also described the enhancement of the antimicrobial effects when appropriate drugs were in vitro associated with DMSO against Rhodococcus equi strains, using the disc diffusion method.

Results observed in present investigation also showed that the in vitro association of EDTATris or DMSO with gentamicin, ciprofloxacin and norfloxacin led to a statiscally significant reduction of MIC values against $P$. aeruginosa strains. Enhancement of the action of some antimicrobials may be explained by the increase in bacterial cell wall permeability in the presence of EDTA-Tris or DMSO, what enables a more consistent penetration of the drugs (Farca et al., 1993; Farca et al., 1997). Additionally, using EDTA-tris compound this synergistic effect may be explained also by the chelation of divalent cations $(\mathrm{Mg}, \mathrm{Ca})$, essentials to the byosynthesis of bacterial peptiodiglycan, leading to damage of cell wall surfaces (Farca et al., 1997).

The present results indicate that the association of EDTA-Tris or DMSO may lead to the in vitro enhancement of the effectiveness of appropriate antimicrobials, which would be useful as additional compounds in intramammary antimicrobial therapy in bovine mastitis caused by $P$. aeruginosa, especially when strains refractory to conventional therapy are involved.

Keywords: bovine, mastitis, Pseudomonas aeruginosa, minimal inhibitory concentration

\section{RESUMO}

A concentração inibitória mínima-MIC em 30 estirpes de Pseudomonas aeruginosa isoladas de mastite bovina foi avaliada utilizando o E-test padrão e o método modificado, pela adição de Tris-EDTA e DMSO. Os métodos modificados apresentaram redução significativa da MIC das estirpes utilizando a gentamicina, a ciprofloxacina e a norfloxacina.

Palavras-chave: bovino, mastite, Pseudomonas aeruginosa, concentração inibitória mínima

\section{REFERENCES}

BRAYTON, C.F. Dimethyl sulfoxide: a review. Cornell Vet., v.76, p.61-90, 1986.

FARCA, A.C.; NEBBIA, P.; RE, G. Potentiation of the in vitro activity of some antimicrobial agents against selected Gram-negative bacteria by EDTAtromethamine. Vet. Res. Commun., v.17, p.77-84, 1993.

FARCA, A.M.; PIROMALLI, G.; MAFFEI, F. Potentiating effect of EDTA-T ris on the activity of antibiotics against resistant bacteria associated with otitis, dermatitis and cystitis. J. Small Anim.Pract., v.38, p.243-245, 1997.

KRIEG, N.R.; HOLT, J.G. Bergey's Manual of Systematic Bacteriology. London: Williams \& Wilkin, 1984. V.1, 984p.

NATIONAL COMMITTEE FOR CLINICAL AND LABORATORY STANDARDS - NCCLS. Performance Standards for Antimicrobial
Susceptibility Testing. 9.ed. Pennsylvania, USA, 1999. $104 \mathrm{p}$.

OSBORNE, A.D.; ARMSTRONG, K.; CATRYSSE, N.H. An outbreak of Pseudomonas mastitis in dairy cows. Can. Vet. J., v.22, p.215-217, 1981.

PAUL, S.; BEZBARUAH, R.L.; ROY, M.K. Multiple antibiotic resistance (MAR) index and its reversion in Pseudomonas aeruginosa. Letters Appl. Microbiol., v.24, p.169-171, 1997.

RADOSTITS, O.M.; BLOOD, D.C.; GAY, C.C. Veterinary Medicine: A textbook of the diseases of cattle, sheep, pigs, goats and horses. 8.ed. Philadelphia: Baillière Tindall, 2000. p.881-884.

RIBEIRO, M.G.; CARVALHO FILHO, A.S.; LISTONI, F.J.P. Dimetilsulfóxido-DMSO no teste de sensibilidade microbiana in vitro em cepas de Rhodococcus equi isoladas de afecções pulmonares em potros. Ciênc. Rural, v.31, p.889-892, 2001.

TRIOLA, M.F. Introdução à estatística. 7.ed. Rio de Janeiro: LTC, 1999. 